\title{
Pigmented Bowen's disease
}

\author{
Amanda Nascimento Cavalleiro de Macedo Mota ${ }^{1}$ \\ Maria de Fatima Guimarães Scotelaro Alves ${ }^{1}$ \\ Carlos Baptista Barcaui ${ }^{1}$
}

\author{
Juan Piñeiro-Maceira ${ }^{1}$ \\ Mónica Jidid Mateus Tarazona ${ }^{1}$
}

\section{DOI: http://dx.doi.org/10.1590/abd1806-4841.20142725}

Abstract: Pigmented Bowen's disease is rare, though more prevalent in men. It presents as a well-delineated plaque in areas unexposed to sun. There are reports of association with seborrheic keratosis, solar lentigo or exuberant pigmentation of genital and intertriginous regions. A specific dermoscopy finding is the presence of brown or gray dots in regular arrangement and coiled or dotted vessels. Thus, we aim to raise awareness of the diagnosis of pigmented Bowen's disease in pigmented lesions.

Keywords: Bowen's disease; Dermoscopy; Skin abnormalities; Skin neoplasms

\section{INTRODUCTION}

Pigmented Bowen's disease is rare, with a higher prevalence in men and blacks aged 60-70 years. It presents clinically as a well-delineated plaque, pigmented, flat and asymptomatic, in areas unexposed to sun. The exact cause of pigmentation remains unclear. This article aims to highlight the possibility of this diagnosis when faced with a pigmented lesion and the dermoscopic features observed in this uncommon form of Bowen's disease.

\section{CASE REPORT}

A 72-year-old, black male complained about the progressive growth of a pigmented and asymptomatic lesion on the left trunk that started eight months before (Figure 1). It appeared clinically as a well-defined plaque with a keratotic surface, measuring $3 \mathrm{~cm}$ in its largest diameter, varying in color from brown to black. He denied comorbidities, exposure to arsenic, radiation and immunosuppression.

We proceeded to the dermatoscopic evaluation, in which criteria for melanocytic lesions were noted, such as a pigment network and streaks (Figure 2). However, other features suggesting nonmelanocytic lesion, including well-defined edges, comedolike openings, structures in digital printing form, commonly found in seborrheic keratosis, also appeared, as brown dots on regular arrangement at the periphery of the lesion.

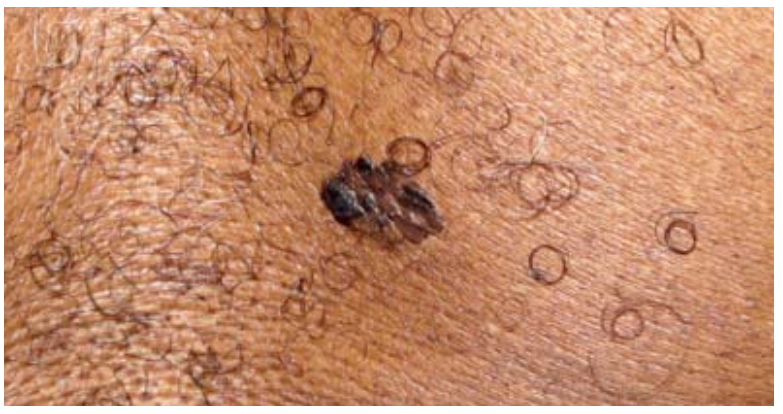

FIGURE 1: A 3cm, black plaque with well-defined edges, heterogeneous and keratotic surface

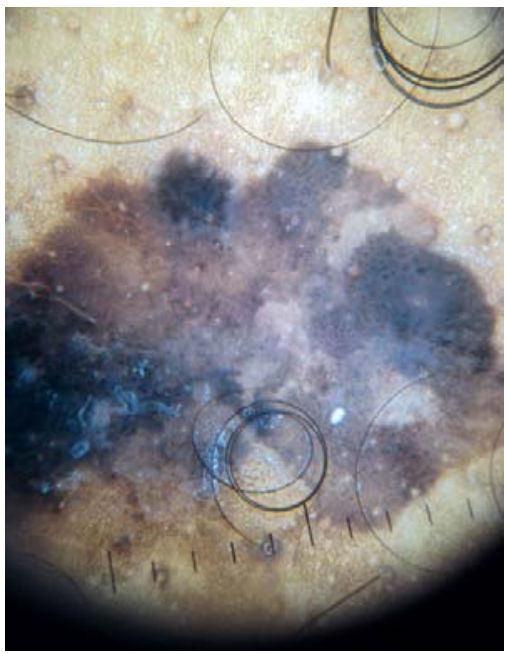

FIGURE 2: Dermoscopy shows asymmetry, well-defined edges with abrupt end, comedo-like openings, fingerprint structures, a pigmented network, radial streaks, amorphous areas with four distinct colors, a blue veil and brown dots in a regular arrangement

Received on 29.04.2013.

Approved by the Advisory Board and accepted for publication on 16.09.2013.

* Work performed at the Hospital Universitário Pedro Ernesto - Universidade do Estado do Rio de Janeiro (HUPE-UERJ) - Rio de Janeiro (RJ), Brasil. Conflict of interest: None

Financial funding: None 
After excision, histopathology revealed hyperkeratosis, acanthosis, inflammatory infiltrate in the upper dermis and vascular ectasia (Figure 3), in addition to melanophages and atypical keratinocytes with pigmented cytoplasm, associated with epidermal hyperproliferation of keratinocytes, thus defining the diagnosis of pigmented Bowen's disease (Figures 4 and 5).

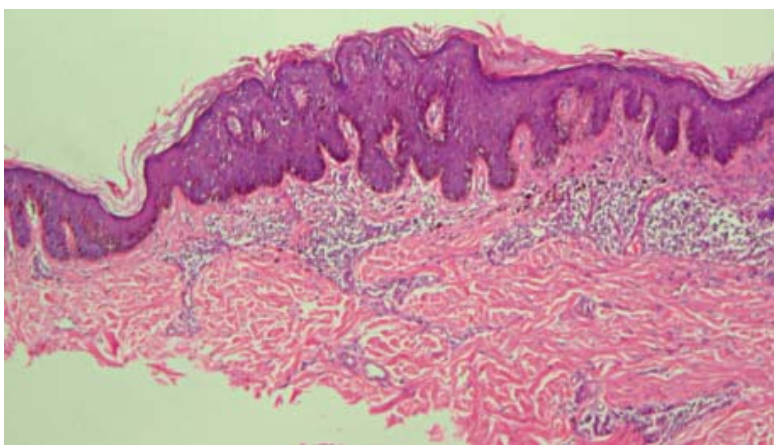

FiguRE 3: Well-defined area of hyperkeratosis, irregular acanthosis and inflammatory infiltrate in the upper dermis $(\mathrm{H} \& \mathrm{E})$

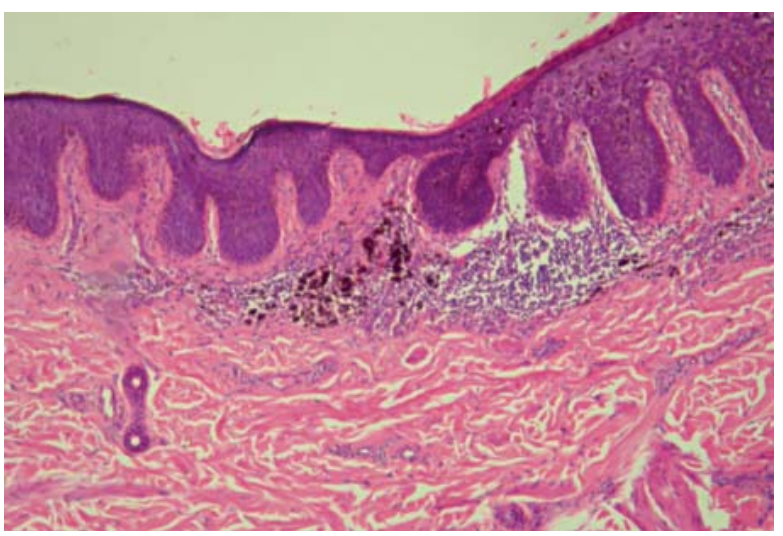

FIGURE 4: Melanophages and melanin pigment in the cytoplasm of atypical keratinocytes ( $\mathrm{H} \& \mathrm{E})$

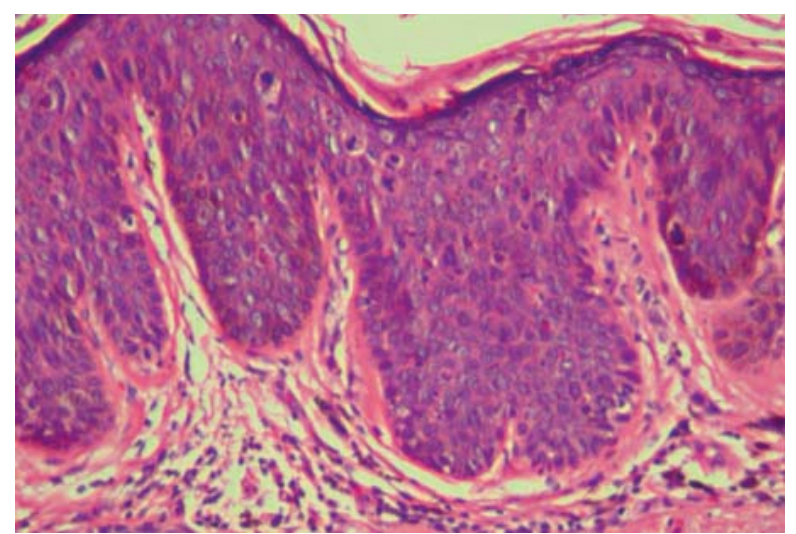

FIGURE 5: Epidermal disorganization due to hyperproliferation of atypical keratinocytes $(\mathrm{H} \& \mathrm{E})$

\section{DISCUSSION}

Bowen's disease or squamous cell carcinoma in situ, appears as an erythematous, well-demarcated, asymptomatic plaque, with slow growth in patients beginning in the sixth decade of life. ${ }^{1}$ In Caucasians, most injuries occur in sun-exposed areas, such as the head, neck and legs. In black patients, it presents atypically, often with preferential involvement of areas unexposed to sun. Factors involved in Bowen's disease include sun exposure, arsenic, infection by the human papilloma virus (HPV), ionizing radiation and trauma. Prolonged exposure to arsenic is considered an etiological factor of lesions in areas unexposed to sun. ${ }^{1}$

According to a review of 420 cases performed by Ragi et al. in 1988, the pigmented variant is rare, occurring in only $1.7 \%$ of cases. ${ }^{2}$ Its prevalence is higher in men and blacks in the seventh decade of life. ${ }^{3}$ It presents clinically as a well-delineated plaque, pigmented, flat and asymptomatic, in areas unexposed to sun. ${ }^{3}$

Histopathological analysis reveals epidermal disarray, atypical keratinocytes and loss of the normal maturation pattern. There is diffuse hyperpigmentation in the epidermis, best observed by Fontana mason staining, melanophages in the papillary dermis and transepidermal elimination of melanin accumulated focally in the stratum corneum. ${ }^{1,4}$

The exact cause of pigmentation remains unclear. This is due to the release of cytokines by atypical keratinocytes, which stimulate the production of melanosomes by melanocytes. Through their dendritic projections, melanosomes are transferred to the cytoplasm of keratinocytes. If present in excessive amounts, this pigment is also deposited in the dermis. ${ }^{4}$ There are reports of association with seborrheic keratosis in $13.6 \%$ of cases, solar lentigo or more exuberant pigmentation of genital and intertriginous regions, found most commonly in sun-protected areas. ${ }^{1}$

Differential diagnoses include melanoma, seborrheic keratosis, solar lentigines, melanocytic nevus, pigmented actinic keratosis, pigmented basal cell carcinoma and bowenoid papulosis. ${ }^{3,4,5}$

In 2004, Argenziano et al. provided the first dermoscopic description of Bowen's disease, highlighting the presence of glomerular or coiled vessels. ${ }^{6}$ In the same year, Zalaudek et al. described the dermoscopic findings of a pigmented lesion $(\mathrm{n}=1)$ as blue homogeneous pigmentation, irregularly distributed bluegray granular structures, pigmented crust and white amorphous areas. ${ }^{7}$

Again in 2004, Stante et al. reported the pigment network findings, brown globules at the periphery and irregular hypopigmented areas similar to regression. ${ }^{8}$ Another review conducted in 2004 by Bugatti et al. yielded 14 cases, revealing multi-component pattern $(100 \%)$, atypical vessels $(87 \%)$, diffuse irregular 
pigmentation $(64 \%)$, irregularly distributed dots and globules $(64 \%)$, focal/multifocal hypopigmentation $(79 \%)$ and surface scales $(64 \%) .{ }^{9}$

In 2010, Cameron et al. described the findings in 52 cases as asymmetry (88\%), amorphous hyperpigmentation pattern alone or in combination with dots, amorphous hypopigmented areas $(67 \%)$, gray or brown dots in a linear arrangement $(21 \%)$ and the presence of coiled or dotted vessels in linear arrangement, as well as a pigment network $(4 \%) .^{10}$

\section{REFERENCES}

1. Marschall SF, Ronan SG, Massa MC. Pigmented Bowen`s disease arising from pigmented seborrheic keratoses. J Am Acad Dermatol. 1990;23:440-4.

2. Ragi G, Turner MS, Klein LE, Stoll HL Jr. Pigmented Bowen`s disease and review of 420 Bowen`s disease lesions. J Dermatol Surg Oncol. 1988;14:765-9.

3. Mora RG, Perniciaro C, Lee B. Cancer of the skin in blacks. III. A review of nineteen black patients with Bowen`s disease. J Am Acad Dermatol. 1984;11:557-62.

4. Vries KD, Lelie B, Habets WJM. Pigmented Bowen`s disease: A report of two cases. Letter to the Editor. Dermatol Surg. 2011: 1061-63.

5. Ishioka P, Yamada S, Michalany NS, Hirata SH. Dermoscopy of Bowen's disease: pigmented variant on the penis. An Bras Dermatol. 2012;87:482-4.

6. Argenziano G, Zalaudek I, Corona R, Sera F, Cicale L, Petrillo G, et al. Vascular structures in skin tumors, a dermoscopy study. Arch Dermatol. 2004;140:1485-9.

7. Zalaudek I, Argenziano G, Leinweber B, Citarella L, Hofmann-Wellenhof R, Malvehy J, et al. Dermoscopy of Bowen`s disease. Br J Dermatol. 2004;150:1112-6.

8. Stante M, de Giorgi V, Massi D, Chiarugi A, Carli P. Pigmented Bowen's disease mimicking cutaneous melanoma: clinical and dermoscopic aspects. Dermatol Surg. 2004;30:541-4.

9. Bugatti L, Filosa G, De Angelis R. Dermoscopic observation of Bowen's disease. J Eur Acad Dermatol Venereol. 2004;18:572-4.

10. Cameron A, Rosendahl C, Tschandl P, Riedl E, Kittler H. Dermatoscopy of pigmented Bowen`s disease. J Am Acad Dermatol. 2010;62:597-604.
Hence, we aim to raise awareness of the diagnosis of pigmented Bowen's disease in hyperpigmented lesions, especially in blacks, even without keratosis or scales. ${ }^{4}$ The most sensitive dermoscopic findings are particularly important, such as amorphous brownish (if there is epidermal melanin), or grayish (if there is dermal melanin) pigmentation in addition to the more specific findings, which include brown dots in a regular arrangement at the periphery of the lesion and dotted or coiled vessels in a linear arrangement..$^{10}$

\author{
MAILING ADDRESS: \\ Amanda Nascimento Cavalleiro de Macedo Mota \\ Boulevard Vinte e oito de setembro, 77 - Vila Isabel \\ 20551-030 - Rio de Janeiro - RJ \\ Brazil \\ E-mail:amanda_mota@hotmail.com
}

How to cite this article: Mota ANCM, Piñeiro-Maceira J, Alves MFGS, Tarazona MJM, Barcaui CB. Pigmented Bowen's disease. An Bras Dermatol. 2014;89(5):825-7. 